\title{
A fully integrated digital LDO with voltage peak detecting and push-pull feedback loop control
}

\author{
Chengtuo Liang ${ }^{1,2 a)}$, Liping Liang ${ }^{1}$, and Zhijun Wang ${ }^{1}$ \\ ${ }^{1}$ Institute of Microelectronics Chinese Academy of Sciences, \\ Beijing 100029, China \\ ${ }^{2}$ School of Microelectronics, University of Chinese Academy of Sciences, \\ Beijing 100029, China
}

a)liangchengtuo@ime.ac.cn

Abstract: A push-pull multi-loop architecture for the digital low drop-out (D-LDO) regulators is presented with small variations of output voltage and $200 \mathrm{~mA}$ load capacity. The propose D-LDO adopts voltage peak detector (VPD) to observe the output voltage ripples. Once undershoot or overshoot on output voltage is detected, the push-pull feedback loop is quickly triggered, which minimizes the voltage shoots even if the load current changes abruptly. Meanwhile, the shift register (S/R) feedback loop regulates the output voltage to desired value with high accuracy. Hence the D-LDO recovers steady state with greatly small voltage spikes. The proposed D-LDO is designed and simulated in SMIC $65 \mathrm{~nm}$ CMOS process with a $0.42 \mathrm{~mm}^{2}$ active area. The simulated voltage overshoot and undershoot are 27 and $26 \mathrm{mV}$ respectively, with load step of 20 to $200 \mathrm{~mA}$ with a 10 -ns edge time. The max load current and quiescent current are $200 \mathrm{~mA}$ and $400 \mu \mathrm{A}$, respectively, and the peak current efficiency is $99.8 \%$.

Keywords: low drop-out (LDO) regulator, voltage peak detector (VPD), shift register (S/R), push-pull feedback loop

Classification: Integrated circuits

\section{References}

[1] J. Liu and N. Maghari: "A fully-synthesizable 0.6 V digital LDO with dualloop control using digital standard cells," NEWCAS Dig. Tech. Papers (2016) 1 (DOI: 10.1109/NEWCAS.2016.7604791).

[2] X. Qu, et al.: "A low-power on-chip LDO with advanced reference buffer," IEICE Electron. Express 11 (2014) 20140824 (DOI: 10.1587/elex.11. 20140824).

[3] S. Maji, et al:: "Digital LDO with time-interleaved comparators for fast response and low ripple,” ISVLSI Dig. Tech. Papers (2016) 337 (DOI: 10. 1109/ISVLSI.2016.137).

[4] M. Cheah, et al: “A 100-mA, 99.11\% current efficiency, 2-mVpp ripple digitally controlled LDO with active ripple suppression," IEEE Trans. Very Large Scale Integr. (VLSI) Syst. 25 (2017) 696 (DOI: 10.1109/TVLSI.2016. 
2596708).

[5] M. Huang and Y. Lu: "A fully integrated digital LDO with coarse-fine-tuning and burst-mode operation," IEEE Trans. Circuits Syst. II, Exp. Briefs 63 (2016) 683 (DOI: 10.1109/TCSII.2016.2530094).

[6] S. B. Nasir, et al:: "All-digital low-dropout regulator with adaptive control and reduced dynamic stability for digital load circuits," IEEE Trans. Power Electron. 31 (2016) 8293 (DOI: 10.1109/TPEL.2016.2519446).

[7] Y. Kim and P. Li: "An ultra-low voltage digitally controlled low-dropout regulator with digital background calibration," ISQED Dig. Tech. Papers (2012) 151 (DOI: 10.1109/ISQED.2012.6187488).

[8] Y.-H. Lee, et al.: "A low quiescent current asynchronous digital-LDO with PLL-modulated fast-DVS power management in $40 \mathrm{~nm} \mathrm{SoC} \mathrm{for} \mathrm{MIPS}$ performance improvement," IEEE J. Solid-State Circuits 48 (2013) 1018 (DOI: 10.1109/JSSC.2013.2237991).

[9] Y. C. Chu and L. R. Chang-Chien: "Digitally controlled low-dropout regulator with fast-transient and autotuning algorithms," IEEE Trans. Power Electron. 28 (2013) 4308 (DOI: 10.1109/TPEL.2012.2230025).

[10] K. Otsuga, et al.: “An on-chip $250 \mathrm{~mA} 40 \mathrm{~nm}$ CMOS digital LDO using dynamic sampling clock frequency scaling with offset-free TDC-based voltage sensor," ISOCC Dig. Tech. Papers (2012) 11 (DOI: 10.1109/SOCC.2012. 6398369).

[11] Y.-J. Lee, et al.: "A 200-mA digital low drop-out regulator with coarse-fine dual loop in mobile application processor," IEEE J. Solid-State Circuits $\mathbf{5 2}$ (2017) 64 (DOI: 10.1109/JSSC.2016.2614308).

[12] E. Alon and M. Horowitz: "Integrated regulation for energy-efficient digital circuits," IEEE J. Solid-State Circuits 43 (2008) 1795 (DOI: 10.1109/JSSC. 2008.925403).

[13] S. Gangopadhyay, et al:: "Modeling and analysis of digital linear dropout regulators with adaptive control for high efficiency under wide dynamic range digital loads," Design Automation Test Europe Conf. (2014) 68 (DOI: 10.7873/ DATE.2014.160).

[14] Y. H. Woo, et al:: "A full-load hybrid compensated LDO with output capacitance range of 0 to $1 \mu \mathrm{F}$," EDSSC Dig. Tech. Papers (2017) 2 (DOI: 10. 1109/EDSSC.2017.8126398).

\section{Introduction}

Systems-on-Chips (SoCs) integrating many individual blocks (such as memory, digital circuits, analog-to-digital converters among others) becomes more widely used in different applications. Power management circuit likes LDO is becoming critical to provide a clean and regulated power supply for each individual block in SoCs [1]. LDO can be classified into analog LDO (A-LDO) [2] and digital LDO (D-LDO) [3, 4]. Digital LDOs are gaining more attention since they have the advantages of suitable for low operating voltages [3], easy integration, small size, programmability, high stability over a wide range of load current variations and a lower sensitivity to process variations [4]. A baseline discrete-time digital LDO usually consists of a comparator, a serial-in parallel-out bidirectional shift register (S/R), and a P-channel MOSFET (PMOS) array acting as the power transistors [5]. In these discrete-time digital LDOs, $\mathrm{S} / \mathrm{R}$ shifts the control signal according to the result of voltage comparator to turn on/off PMOS transistors and regulates the 
output voltage with a master clock. Thus the generation and propagation of control signal will be synchronous. Due to their synchronous and sequential nature of switching, the baseline digital LDOs suffer from slower transient responses to large load current steps resulting in a large voltage undershoot/overshoot and show a tradeoff between steady-state stability and transient response [6].

Several previous works have been proposed to tackle this issue. A gated voltage controlled oscillator (GVCO) with counters is used in [7] to provide more "continuous" information of the digitalized output voltage level. Based on that information, the D-LDO in [7] controls multiple power transistors concurrently and the transient performance is improved. However, the inherent sensitivity to PVT variations limits its applications. Asynchronous control and adaptive sizing that changes resolution are applied in the D-LDO, targeting at a balance in high speed and fine resolution in [8]. Similarly to [7], the PVT variations become a headache too. The D-LDO presented in [9] using a SAR-type ADC and a separate DAC driving a single power transistor. But, this design is not power-efficient since its complex design consumes additional quiescent current. Another work shown in [10] adopts a TDC-based 4-b ADC and a PID controller for stability compensation. In that design, the performance of fast transient response is achieved through dynamic clock scaling from normally $250 \mathrm{MHz}$ to $1 \mathrm{GHz}$. However, it consumes large quiescent current of $2.5 \mathrm{~mA}$. To trade-off between transition response and steady performance, the coarse-fine tuning technique is widely used such as in $[5,11]$. Briefly, coarse-fine tuning technique mainly includes two aspects, namely the coarse-tuning and fine-tuning. The coarse-tuning with more power MOS units and high sampling frequency enhances transient performance while the fine-tuning with small power MOS units and slow sampling frequency achieves high steady and low quiescent current. Unfortunately, the coarse/fine-turning algorithm leads to complexity of controlled block.

In this paper, a novel all digital LDO (D-LDO) with voltage peak detecting and push-pull feedback loops is presented. The proposed D-LDO can achieve small voltage spikes, low quiescent current, and up to $200-\mathrm{mA}$ load current capacity. This brief is organized as follows. The architecture and core circuit of our proposed D-LDO is illustrated in Section 2. Steady and small signal model analysis is presented in Section 3. Simulation results are shown in Section 4. Finally, conclusions are made in Section 5.

\section{Implementation and design considerations}

\subsection{Architecture of proposed D-LDO}

The architecture of the proposed digital LDO is presented in Fig. 1. Push-pull topology as in [12] is used for multi-loop operation. Generic digital LDO is comprised of load RC network, clock comparator $\mathrm{CMP}_{2}$, shift register $\mathrm{S} / \mathrm{R}$ and P-channel MOSFET (PMOS) array $\left(\mathrm{PG}_{0}\right.$ to $\left.\mathrm{PG}_{159}\right)$ acting as the power transistors. In the proposed architecture, a push-pull feedback loop trigger (PP-FLT) scheme is embedded in the generic digital LDO to control the push-pull output stage that consists of PMOS array $\left(\mathrm{PG}_{0}\right.$ to $\left.\mathrm{PG}_{159}\right)$ and N-channel MOSFET (NMOS) transistor $\mathrm{N}_{\mathrm{G}}$. As shown in Fig. 1, there are three feedback loops in the proposed 
architecture, namely $\mathrm{L}_{\mathrm{SR}}, \mathrm{L}_{\mathrm{P}}$ and $\mathrm{L}_{\mathrm{N}}$. The shift register $(\mathrm{S} / \mathrm{R})$ feedback loop $\mathrm{L}_{\mathrm{SR}}$ that includes load RC network, clock comparator $\mathrm{CMP}_{2}$, shift register $\mathrm{S} / \mathrm{R}$ and the PMOS array is widely used in generic D-LDO to regulate the output voltage to a desired value under difference load conditions, while the proposed push-pull feedback loops $\mathrm{L}_{\mathrm{P}}$ and $\mathrm{L}_{\mathrm{N}}$ consisting of load RC network, PP-FLT scheme and the push-pull output stage are employed to reduce the variations of $\mathrm{V}_{\text {OUT }}$.

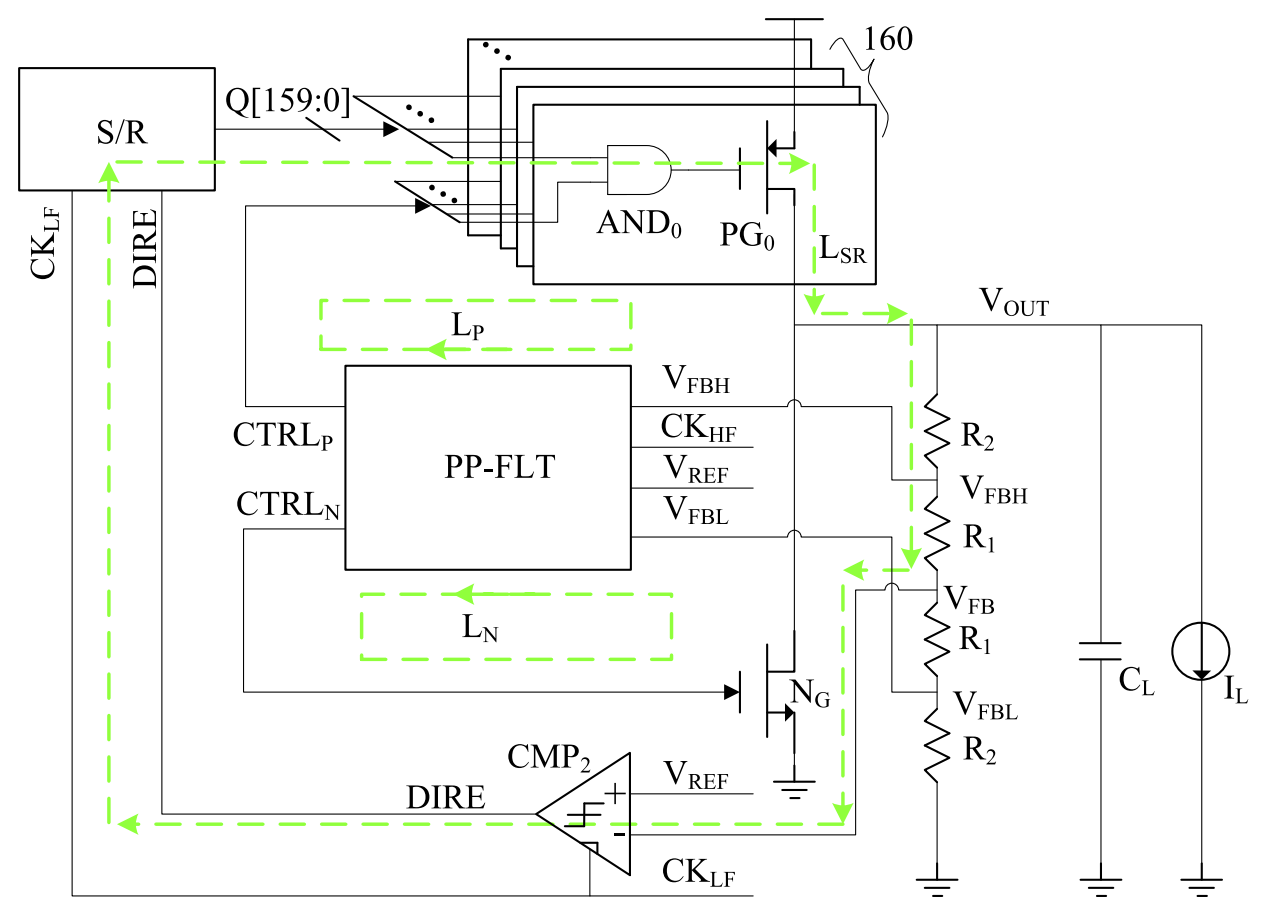

Fig. 1. Architecture of proposed D-LDO with voltage peak detecting and push-pull feedback loop control.

The undershoot and overshoot detection boundaries are $\left(2 \mathrm{~V}_{\mathrm{REF}}-\Delta \mathrm{V}_{\mathrm{L}}\right)$ and $\left(2 V_{R E F}+\Delta V_{H}\right)$, where $2 V_{R E F}$ denotes the desired value of $V_{O U T}$ in steady state, $\Delta \mathrm{V}_{\mathrm{L}}$ is the predetermined undershoot detection threshold, and $\Delta \mathrm{V}_{\mathrm{H}}$ is the overshoot detection threshold. When $\mathrm{V}_{\mathrm{OUT}}$ is within the range between $\left(2 \mathrm{~V}_{\mathrm{REF}}-\Delta \mathrm{V}_{\mathrm{L}}\right)$ and $\left(2 \mathrm{~V}_{\mathrm{REF}}+\Delta \mathrm{V}_{\mathrm{H}}\right)$, the D-LDO works only at $\mathrm{L}_{\mathrm{SR}}$-turning mode, at which only shift register $(\mathrm{S} / \mathrm{R})$ feedback loop $\mathrm{L}_{\mathrm{SR}}$ is available to ensure that $\mathrm{V}_{\mathrm{OUT}}$ incessantly tracks the desired voltage. On the other hand, once $V_{\text {OUT }}$ exceeds undershoot or overshoot detection boundaries $\left(2 \mathrm{~V}_{\mathrm{REF}}-\Delta \mathrm{V}_{\mathrm{L}}\right.$ or $\left.2 \mathrm{~V}_{\mathrm{REF}}+\Delta \mathrm{V}_{\mathrm{H}}\right)$, the $\mathrm{L}_{\mathrm{P}}$-turning or $\mathrm{L}_{\mathrm{N}}$-turning mode is quickly triggered while $\mathrm{L}_{\mathrm{SR}}$-turning mode is always available. In this scenario, the push-pull feedback loop $\mathrm{L}_{\mathrm{P}} / \mathrm{L}_{\mathrm{N}}$ is enable to prevent a large undershoot/overshoot on $\mathrm{V}_{\text {OUT }}$ while the $\mathrm{L}_{\mathrm{SR}}$ regulates the $\mathrm{V}_{\text {OUT }}$ to $2 \mathrm{~V}_{\mathrm{REF}}$ with a desired load current.

To achieve wide bandwidths of push-pull feedback loops $\left(\mathrm{L}_{\mathrm{P}}, \mathrm{L}_{\mathrm{N}}\right)$, a high frequency $(1 \mathrm{GHz})$ clock $\left(\mathrm{CK}_{\mathrm{HF}}\right)$ is employed in the PP-FLT scheme, in addition, the push-pull output stage is directly controlled by PP-FLT scheme. Though a high frequency clock is needed, it is only used to trigger two clock comparators in PP-FLT scheme. Thus the increment of power consumption caused by the high 
steady state, the D-LDO operates only on the $\mathrm{L}_{\mathrm{SR}}$-turning mode, with a small power MOS strength and low frequency $(50-\mathrm{kHz})$ clock $\left(\mathrm{CK}_{\mathrm{LF}}\right)$. Therefore, with the proposed PP-FLT scheme, the D-LDO could effectively improve transient response and achieve low quiescent current simultaneously.

\subsection{Push-pull feedback loop trigger (PP-FLT) scheme}

As shown in Fig. 2, the PP-FLT scheme includes a voltage peak detector (VPD), a 2-input OR gate, a 2-input INAND gate and a 2-input AND gate, whereas VPD is composed of 2 clock comparators $\left(\mathrm{CMP}_{0}, \mathrm{CMP}_{1}\right)$ and a 2-input XNOR gate. The VPD is used to detect the undershoot/overshoot on $V_{\text {OUT }}$, which outputs 1 if $V_{\text {OUT }}$ is not within the range between $\left(2 \mathrm{~V}_{\mathrm{REF}}-\Delta \mathrm{V}_{\mathrm{L}}\right)$ and $\left(2 \mathrm{~V}_{\mathrm{REF}}+\Delta \mathrm{V}_{\mathrm{H}}\right)$. With the output of VPD (PPFL $\mathrm{EN}_{\mathrm{E}}$ ) becoming high, the signal DIRE $\mathrm{HF}_{\mathrm{HF}}$ generated by the OR gate indicates whether undershoot on $\mathrm{V}_{\text {OUT }}$ was detected or overshoot. Then signals PPFL $_{E N}$ and DIRE $E_{\mathrm{HF}}$ are fed to the INAND gate as well as AND gate to generate the control signals CTRL $L_{P}$ and $C T R L_{N}$. The signals $C T R L_{P}$ and $C T R L_{N}$ determine which feedback loop of $\mathrm{L}_{\mathrm{P}}$ and $\mathrm{L}_{\mathrm{N}}$ will be triggered after undershoot/overshoot on $\mathrm{V}_{\text {OUT }}$ has been detected.

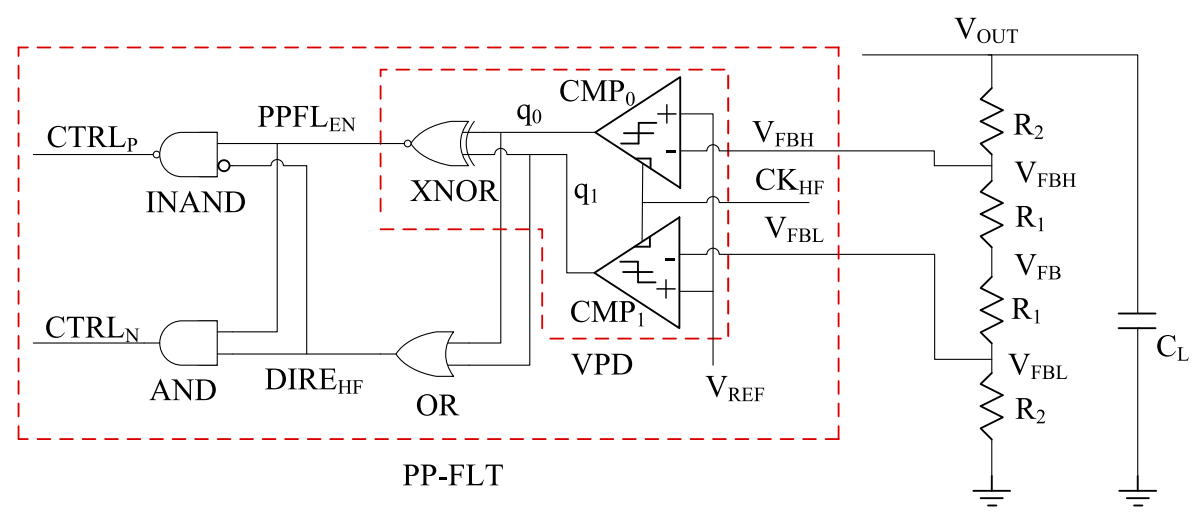

Fig. 2. Proposed push-pull feedback loop trigger (PP-FLT) scheme with voltage peak detector (VPD).

The operation processes of PP-FLT scheme are given in Fig. 3. In the first case [see Fig. 3(a)], under certain disturbances, the output voltage decreases by $\Delta V_{L}$, resulting in a variation $\Delta \mathrm{V}_{\mathrm{FBH}}$ of feedback voltage $\mathrm{V}_{\mathrm{FBH}}$. When the $\mathrm{V}_{\mathrm{FBH}}$ drops below reference voltage $\mathrm{V}_{\mathrm{REF}}$, the output of $\mathrm{CMP}_{0}$ (denoted as $\mathrm{q}_{0}$ ) changes from 1 to 0 while the output of $\mathrm{CMP}_{1}$ (marked by $\mathrm{q}_{1}$ ) remains constant 0 since the $\mathrm{V}_{\mathrm{FBL}}$ is always below $\mathrm{V}_{\mathrm{REF}}$. Once $\mathrm{q}_{0}$ becomes low, the output of VPD (illustrated as PPFL $_{E N}$ ) flips from 0 to 1 while signal DIRE $_{\mathrm{HF}}$ changes to 0 , indicating that undershoot on $\mathrm{V}_{\text {OUT }}$ has been detected. Then, the signal CTRL $L_{P}$ becomes 0 , which will trigger the push pull feedback loop $L_{P}$ to suppress the undershoot voltage. The process of deriving $\Delta \mathrm{V}_{\mathrm{L}}$ is shown below.

Both of the $\mathrm{V}_{\mathrm{FBL}}$ and $\mathrm{V}_{\mathrm{FBH}}$ come from the load bias resistor ladder consisted of $R_{1}$ and $R_{2}$, hence $V_{F B H}$ is a function of $V_{\text {OUT }}$, as given by

$$
V_{F B H}=\frac{2 R_{1}+R_{2}}{2\left(R_{1}+R_{2}\right)} V_{O U T}
$$




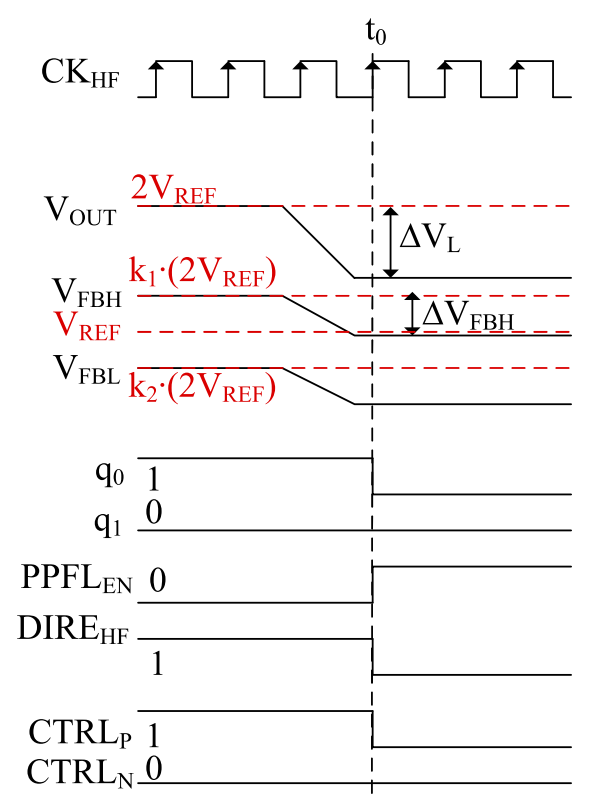

(a)

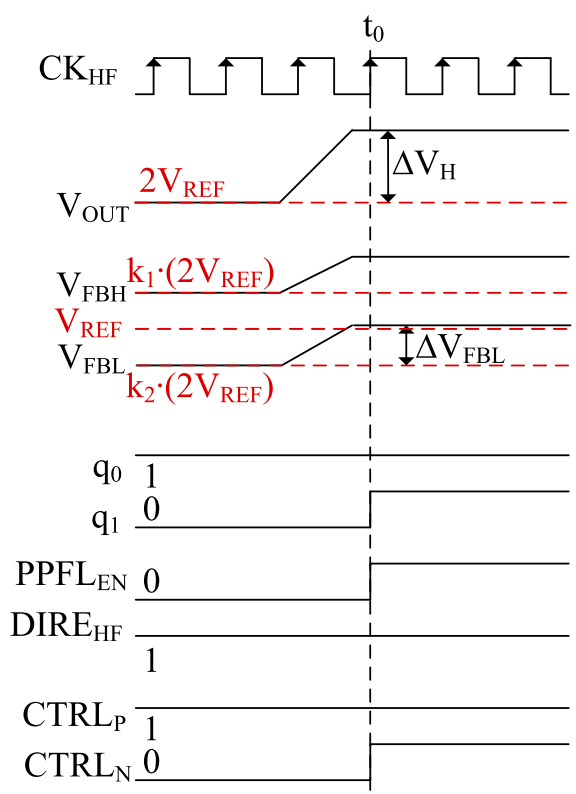

(b)

Fig. 3. Operation processes of the proposed PP-FLT for (a) undershoot and (b) overshoot voltages detection.

At time $\mathrm{t}_{0}$, Eq. (1) is expressed as

$$
V_{F B H} \mid t_{0}=\left(V_{F B H @ S S}-\Delta V_{F B H}\right)=\frac{2 R_{1}+R_{2}}{2\left(R_{1}+R_{2}\right)}\left(V_{\text {OUT @SS }}-\Delta V_{L}\right),
$$

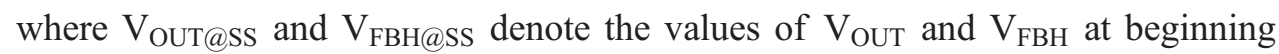
steady state, respectively. The $\mathrm{V}_{\text {OUT@Ss }}$ is given by

$$
V_{O U T @ S S}=2 V_{R E F},
$$

while the $\mathrm{V}_{\mathrm{FBH} @ S \mathrm{~S}}$ is given by

$$
V_{F B H @ S S}=\left(\frac{2 R_{1}+R_{2}}{2\left(R_{1}+R_{2}\right)}\right) V_{\text {OUT@SS }}=\left(\frac{2 R_{1}+R_{2}}{2\left(R_{1}+R_{2}\right)}\right) 2 V_{R E F},
$$

hence $\mathrm{V}_{\mathrm{FBH} @ S s}$ becomes

$$
V_{F B H @ S S}=k_{1}\left(2 V_{R E F}\right),
$$

where

$$
k_{1}=\frac{2 R_{1}+R_{2}}{2\left(R_{1}+R_{2}\right)} .
$$

In order to trigger the push-pull feedback loop $\mathrm{L}_{\mathrm{P}}$, the feedback voltage $\mathrm{V}_{\mathrm{FBH}}$ needs to satisfy the following condition

$$
V_{F B H}<V_{R E F} .
$$

At time $\mathrm{t}_{0}$, Eq. (7) becomes

$$
V_{F B H} \mid t_{0}=\frac{2 R_{1}+R_{2}}{2\left(R_{1}+R_{2}\right)}\left(V_{\text {OUT } @ S S}-\Delta V_{L}\right)<V_{R E F} .
$$

By solving the Eq. (3) and Eq. (8), $\Delta \mathrm{V}_{\mathrm{L}}$ is calculated as

$$
\Delta V_{L}>\frac{1}{2+R_{2} / R_{1}}\left(2 V_{R E F}\right)
$$


The undershoot detection threshold is define as

$$
\Delta V_{L}=\frac{1}{2+R_{2} / R_{1}}\left(2 V_{R E F}\right) .
$$

On the other hand [see Fig. 3(b)], the output voltage $V_{\text {OUT }}$ increases by $\Delta V_{H}$, leading to a variation $\Delta \mathrm{V}_{\mathrm{FBL}}$ of feedback voltage $\mathrm{V}_{\mathrm{FBL}}$. Once the $\mathrm{V}_{\mathrm{FBL}}$ is higher than $V_{R E F}$, the $C T R L_{N}$ thus changes from 0 to 1 , which enables $L_{N}$-turning mode to reduce the overshoot on $\mathrm{V}_{\text {OUT }}$. Similarly to $\mathrm{k}_{1}$, parameter $\mathrm{k}_{2}$ shown in Fig. $3(\mathrm{~b})$ as well as Fig. 3(a) is expressed as

$$
k_{2}=\frac{R_{2}}{2\left(R_{1}+R_{2}\right)} .
$$

Analogously to $\Delta \mathrm{V}_{\mathrm{L}}$, the overshoot detection threshold $\Delta \mathrm{V}_{\mathrm{H}}$ is define as

$$
\Delta V_{H}=\frac{R_{1}}{R_{2}}\left(2 V_{R E F}\right) \text {. }
$$

Therefore the voltage shoot detection thresholds (marked by $\Delta \mathrm{V}_{\mathrm{L}}, \Delta \mathrm{V}_{\mathrm{H}}$ ) are proportional to the $\mathrm{R}_{1} / \mathrm{R}_{2}$. In this Letter, $\mathrm{V}_{\mathrm{REF}}=0.5 \mathrm{~V}, \mathrm{R}_{1}=0.1 \mathrm{~K} \Omega, \mathrm{R}_{2}=4.9 \mathrm{~K} \Omega$ and $\mathrm{R}_{1} / \mathrm{R}_{2}=1 / 49$. Thus $\Delta \mathrm{V}_{\mathrm{L}} \approx 19.6 \mathrm{mV}, \Delta \mathrm{V}_{\mathrm{H}} \approx 20 \mathrm{mV}$.

\subsection{Clocked comparator and S/R}

As given in Fig. 4(a), the clocked comparator scheme introduced in [5] is used in the proposed D-LDO. A latch following the first stage restores the output signal. The comparator operates with two phases, namely pre-charge phase and regenerative phase. When CLK signal is low (pre-charge phase), the internal nodes are pulled to high while the latch outputs keep unchanged; when CLK rises to high (regenerative phase), the comparator accumulates the difference between $\mathrm{V}_{\text {INP }}$ and $\mathrm{V}_{\text {INN }}$ and rapidly amplifies the small difference to full swing, then the output latch will restore the output signal. Table I is the true table of the clocked comparator.

Table I. True table of clocked comparator

\begin{tabular}{c|c}
\hline & CMP OUT \\
\hline $\mathrm{V}_{\text {INP }}<\mathrm{V}_{\text {INN }}$ & "1" \\
\hline $\mathrm{V}_{\text {INP }}>\mathrm{V}_{\text {INN }}$ & "0" \\
\hline
\end{tabular}

Fig. 4(b) shows the scheme of the 160-bit shift register (S/R), which consists of D-type flip-flops and multiplexers. The Q[159:0] are the outputs of S/R, which control the PMOS array $\mathrm{PG}_{159} \sim \mathrm{PG}_{0}$. When DIRE is low, all the bits Q are shifted to the right by one count at the rising edge of the sampling clock CLK and vice versa, indicating one more PMOS transistor to turn on/off.

\subsection{Operation principles}

The operation principles of the proposed D-LDO are depicted in Fig. 5. In steady state, the D-LDO works only at $\mathrm{L}_{\mathrm{SR}}$-turning mode, with a small PMOS transistor step and low frequency clock $\mathrm{CK}_{\mathrm{LF}}$. As shown in Fig. 5(a), when the load current $\left(\mathrm{I}_{\mathrm{L}}\right)$ changes abruptly from 20 to $200 \mathrm{~mA}, \mathrm{~V}_{\text {OUT }}$ starts to drop. The $\mathrm{S} / \mathrm{R}$ feedback 


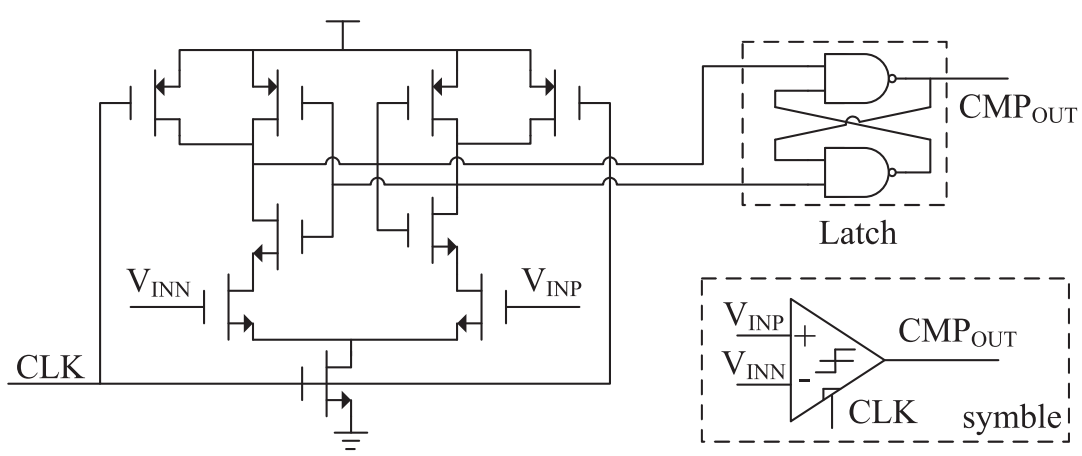

(a)

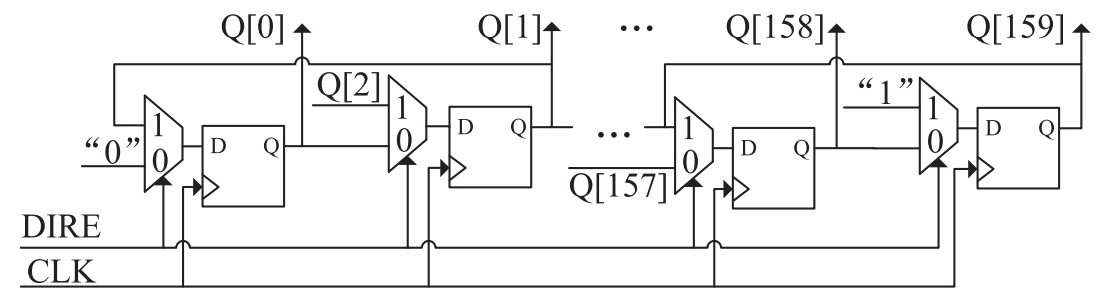

(b)

Fig. 4. Schemes of (a) clocked comparator and (b) shift register.

loop $\left(\mathrm{L}_{\mathrm{SR}}\right)$ senses the $\mathrm{V}_{\mathrm{OUT}}$ drop and generates more logic low control signal among Q[159:0], which will turn on more extra PMOS transistors to suppress $\mathrm{V}_{\text {OUT }}$ drop. However, since the transition speed of $\mathrm{L}_{\mathrm{SR}}$ is quite slow, $\mathrm{V}_{\mathrm{OUT}}$ keep going downward. At time $t_{0}$, when $V_{\text {OUT }}$ exceeds the undershoot detection boundary $\left(2 \mathrm{~V}_{\mathrm{REF}}-\Delta \mathrm{V}_{\mathrm{L}}\right)$, the output of PP-FLT scheme (i.e. CTRL $)$ immediately set to logic 0. So, $\mathrm{L}_{\mathrm{P}}$-turning mode is triggered and all of the PMOS transistors $\left(\mathrm{PG}_{0}\right.$ to $\mathrm{PG}_{159}$ ) are turned on to charge the $\mathrm{C}_{\mathrm{L}}$ with theatrically strength current. Then $\mathrm{V}_{\text {OUT }}$ recovers to the level above $\left(2 \mathrm{~V}_{\mathrm{REF}}-\Delta \mathrm{V}_{\mathrm{L}}\right)$ quickly. As thus, signal CTRL $L_{\mathrm{P}}$ reset to logic 1 disabling $L_{P}$-turning mode at time $t_{1}$, which leads $V_{\text {OUT }}$ to go downward and exceed $\left(2 V_{R E F}-\Delta V_{L}\right)$ once more at time $t_{2}$. Then, $L_{P}$-turning mode is enabled and regulates $\mathrm{V}_{\mathrm{OUT}}$ to the level above $\left(2 \mathrm{~V}_{\mathrm{REF}}-\Delta \mathrm{V}_{\mathrm{L}}\right)$ again. Next, $\mathrm{L}_{\mathrm{P}}$-turning mode is disabled and enabled alternately to limit $\mathrm{V}_{\text {OUT }}$ drop until enough PMOS transistors are turned on by $\mathrm{S} / \mathrm{R}$. Hence the $\mathrm{V}_{\text {OUT }}$ successfully recovers to common level with greatly small undershoots. Finally, $\mathrm{S} / \mathrm{R}$ keeps regulating $\mathrm{V}_{\text {OUT }}$ closer to the desired value and makes the D-LDO enter steady state.

Fig. 5(b) shows the case of load current decreasing abruptly from 200 to $20 \mathrm{~mA}$. Once $\mathrm{V}_{\text {OUT }}$ exceeds the overshoot detection boundary $\left(2 \mathrm{~V}_{\mathrm{REF}}+\Delta \mathrm{V}_{\mathrm{H}}\right)$, the PP-FLT scheme triggers the $\mathrm{L}_{\mathrm{N}}$-turning mode by setting $\mathrm{CTRL}_{\mathrm{N}}$ to 1 , which turns on the NMOS transistor $\mathrm{N}_{\mathrm{G}}$ to discharge the $\mathrm{C}_{\mathrm{L}}$ with strength current, hence, $\mathrm{V}_{\text {OUT }}$ immediately drops to below level of $\left(2 \mathrm{~V}_{\mathrm{REF}}+\Delta \mathrm{V}_{\mathrm{H}}\right)$. To limit the overshoots on $\mathrm{V}_{\text {OUT }}$, the $\mathrm{L}_{\mathrm{N}}$-turning mode toggles between on and off alternatively depending on whether $\mathrm{V}_{\text {OUT }}$ exceeds $\left(2 \mathrm{~V}_{\mathrm{REF}}+\Delta \mathrm{V}_{\mathrm{H}}\right)$ or not. Simultaneously, $\mathrm{L}_{\mathrm{SR}}$-turning mode is available and $\mathrm{S} / \mathrm{R}$ generates more logic high control signal among $\mathrm{Q}$ [159:0] to turn off extra PMOS transistors. After most of the extra PMOS transistors have been turned off, $\mathrm{L}_{\mathrm{N}}$-turning mode is not need to be triggered any more. Finally, D-LDO continues to work at $\mathrm{L}_{\mathrm{SR}}$-turning mode which adjusts $\mathrm{V}_{\mathrm{OUT}}$ closer to $2 \mathrm{~V}_{\mathrm{REF}}$ and steady state is hence achieved. 


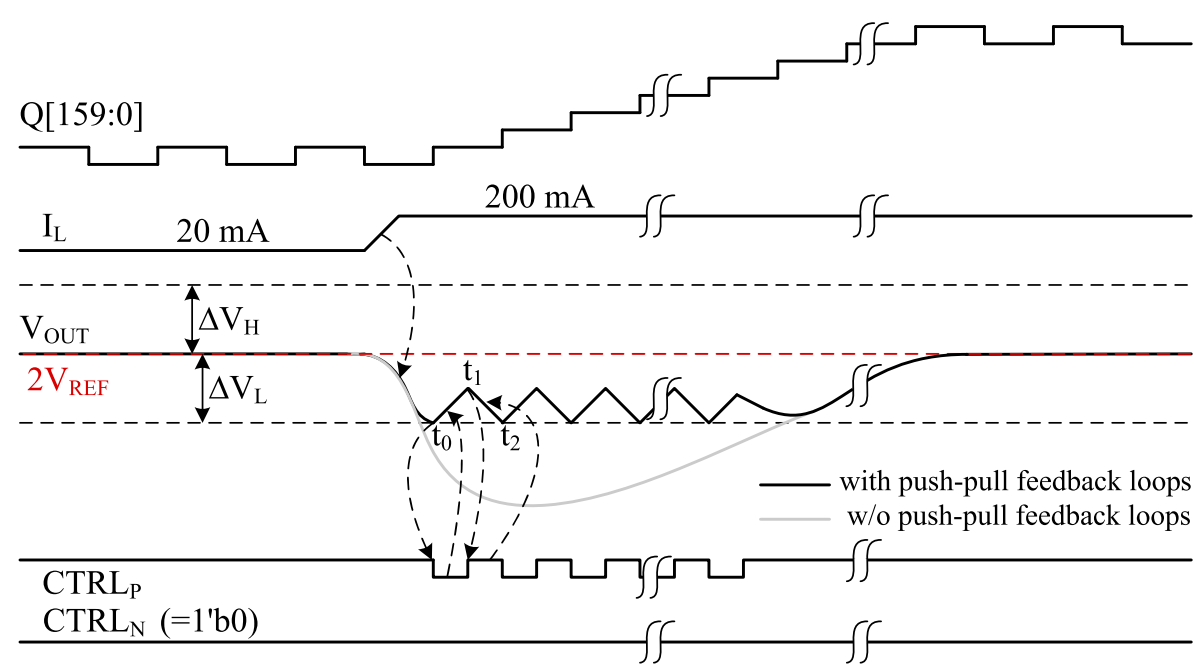

(a)

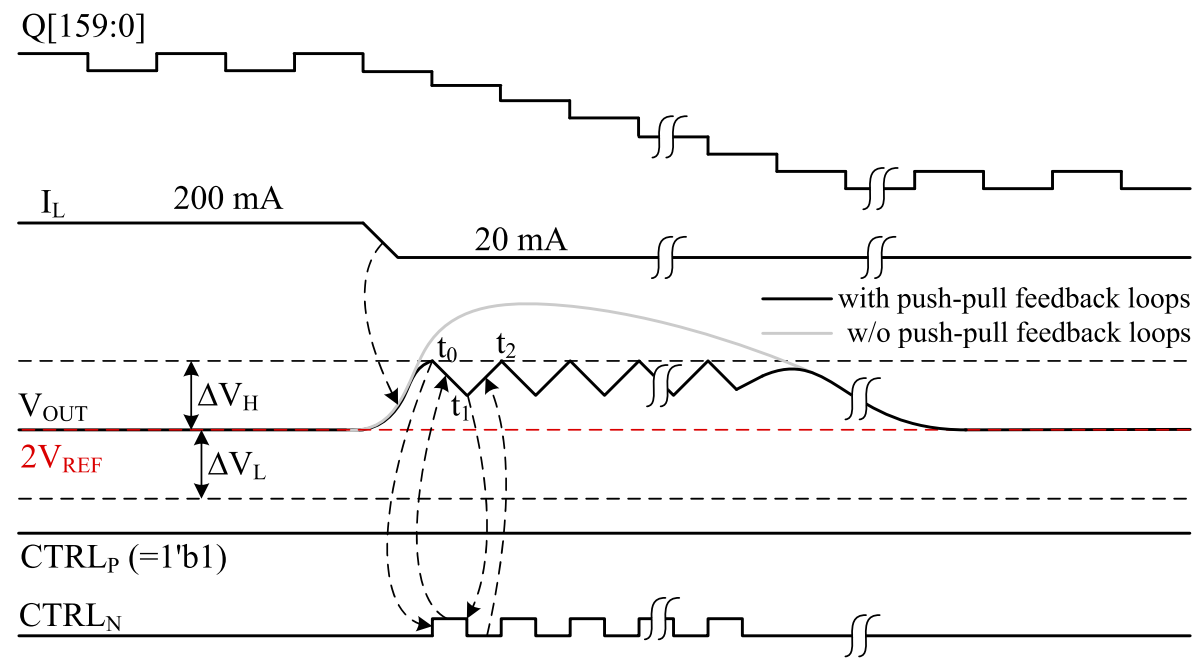

(b)

Fig. 5. Transient responses of proposed D-LDO with (a) positive and (b) negative load current step.

These operations prevent large spikes on $\mathrm{V}_{\text {OUT }}$ and significantly improve transition response.

\section{Steady analyses}

Fig. 6 is the illustration of unsteady push-pull feedback loop control. The region from $t_{0}$ to $t_{1}$ depicts that all PMOS transistors are turned on at time $t_{0}$ to charge $C_{L}$ with charged current $\mathrm{I}_{\mathrm{PGMAX}}$ and pull $\mathrm{V}_{\mathrm{OUT}}$ up. However, since either the $\mathrm{C}_{\mathrm{L}}$ is too small or $\mathrm{I}_{\text {PGMAX }}$ is too strong, $\mathrm{V}_{\text {OUT }}$ quickly goes upward to $\left(2 \mathrm{~V}_{\mathrm{REF}}+\Delta \mathrm{V}_{\mathrm{H}}\right)$ within a period of $\mathrm{CK}_{\mathrm{HF}}$. At time $\mathrm{t}_{1}$, the D-LDO directly toggles from $\mathrm{L}_{\mathrm{P}}$-turning mode to $\mathrm{L}_{\mathrm{N}}$-turning mode without entering steady state. Hence, with $\mathrm{L}_{\mathrm{P}}$ releasing control of PMOS array, NMOS transistor $\mathrm{N}_{\mathrm{G}}$ is turned on immediately by feedback loop $\mathrm{L}_{\mathrm{N}}$ to discharge $\mathrm{C}_{\mathrm{L}}$ with discharged current $\mathrm{I}_{\mathrm{NGMAX}}$, leading to $\mathrm{V}_{\mathrm{OUT}}$ goes downward. Unfortunately, the $\mathrm{V}_{\mathrm{OUT}}$ unexpectedly drops to $\left(2 \mathrm{~V}_{\mathrm{REF}}-\Delta \mathrm{V}_{\mathrm{L}}\right)$ at next clock rise edge (namely at time $t_{2}$ ) leading to $L_{P}$-turning mode be enable again. As thus, $C_{L}$ is charged and discharged alternatively within adjacent clock period of $\mathrm{CK}_{\mathrm{HF}}$. $\mathrm{V}_{\mathrm{OUT}}$ 
thus oscillates between $\left(2 \mathrm{~V}_{\mathrm{REF}}-\Delta \mathrm{V}_{\mathrm{L}}\right)$ and $\left(2 \mathrm{~V}_{\mathrm{REF}}+\Delta \mathrm{V}_{\mathrm{H}}\right)$ with a period of $2 \mathrm{~T}_{\mathrm{HF}}$, resulting in unsteady problems. To avoid that unwanted unsteady state, the variation of charged and discharged voltage $\left(\Delta \mathrm{V}_{\mathrm{CL}}\right)$ within a period $\left(\mathrm{T}_{\mathrm{HF}}\right)$ of $\mathrm{CK}_{\mathrm{HF}}$ should be smaller than $\left(\Delta \mathrm{V}_{\mathrm{L}}+\Delta \mathrm{V}_{\mathrm{H}}\right)$. Hence the steady conditions of push-pull feedback loop control are given as follows

$$
\begin{aligned}
& \frac{I_{P G M A X} T_{H F}}{C_{L}} \leq\left(\Delta V_{L}+\Delta V_{H}\right) \\
& \frac{I_{N G M A X} T_{H F}}{C_{L}} \leq\left(\Delta V_{L}+\Delta V_{H}\right),
\end{aligned}
$$

where $\mathrm{I}_{\mathrm{PGMAX}}$ and $\mathrm{I}_{\mathrm{NGMAX}}$ are maximum current available to charge and discharge the $C_{L}$, respectively. The value of $C_{L}$ can be given by solving Eq. (13) and Eq. (14).

$$
C_{L} \geq \max \left\{\left(\frac{I_{P G M A X} T_{H F}}{\Delta V_{L}+\Delta V_{H}}\right),\left(\frac{I_{N G M A X} T_{H F}}{\Delta V_{L}+\Delta V_{H}}\right)\right\} .
$$

In the proposed D-LDO, the $\mathrm{I}_{\mathrm{PGMAX}}$ and $\mathrm{I}_{\mathrm{NGMAX}}$ are approximately $200 \mathrm{~mA}, \mathrm{~T}_{\mathrm{HF}}$ is $1 \mathrm{~ns}$, and $\left(\Delta \mathrm{V}_{\mathrm{L}}+\Delta \mathrm{V}_{\mathrm{H}}\right)$ is approximately $40 \mathrm{mV}$. Thus $\mathrm{C}_{\mathrm{L}}$ becomes as follows

$$
C_{L} \geq 5 \mathrm{nF}
$$

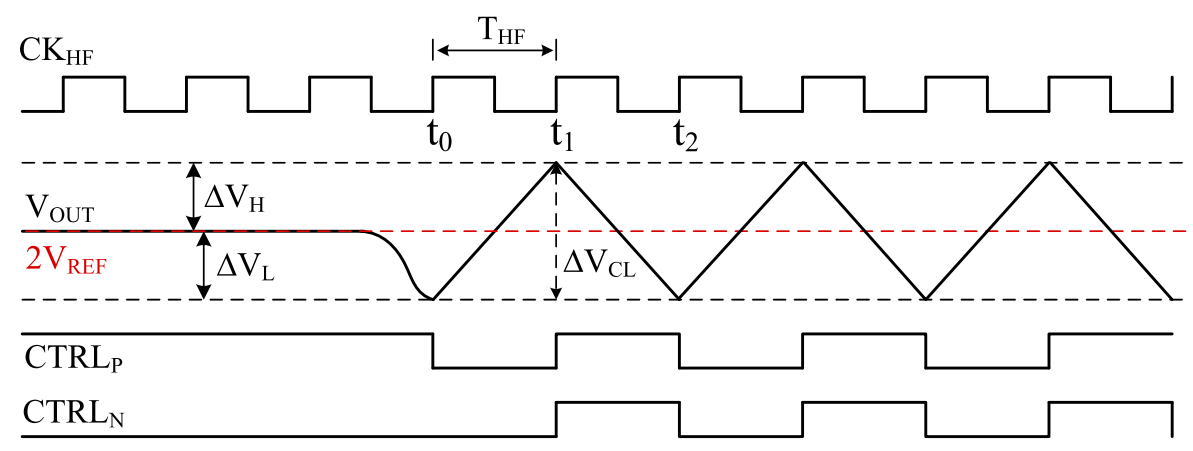

Fig. 6. Illustration of unsteady push-pull feedback loop control.

The small signal model of $\mathrm{S} / \mathrm{R}$ feedback loop $\mathrm{L}_{\mathrm{SR}}$ in the proposed $\mathrm{D}-\mathrm{LDO}$ is shown in Fig. 7(a) which is made up of a sampled comparator $\mathrm{CMP}_{2}$, a shift register $(\mathrm{S} / \mathrm{R})$, a zero-order hold $(\mathrm{ZOH})$, and a first-order plant representing the continuous time plant consisting of the PMOS transistor array and the RC load. A feedback coefficient $\beta=1 / 2$ has been inserted into the feedback loop because the feedback voltage $\mathrm{V}_{\mathrm{FB}}$ is generated by the load bias resistor ladder and $\mathrm{V}_{\mathrm{FB}}=$ $1 / 2 \mathrm{~V}_{\text {OUT }}$. The open-loop transfer function $\mathrm{G}(\mathrm{z})$ is written as Eq. (17) according to $[13]$

$$
G(z)=K_{D C}\left(1-e^{-\alpha T}\right) \frac{z}{(z-1)\left(z-e^{-\alpha T}\right)},
$$

where $K_{D C}$ is the loop $D C$ gain, $\alpha$ is the time constant of the output pole, and $\mathrm{T}$ is the sampling time interval of $S / R$. In the synchronous $S / R$ feedback loop, sampling time interval $\mathrm{T}$ equals to the period of $\mathrm{CK}_{\mathrm{LF}}$ which is $20 \mu \mathrm{s}$. Since the feedback coefficient $\beta=1 / 2$, the overall closed loop transfer function of the digital 


$$
H(z)=G(z) /\left(1+\frac{1}{2} G(z)\right) .
$$

Eq. (18) provides insight into the stability of the proposed D-LDO. The root loci of this system can be plotted in Fig. 7(b). All of the poles in z-domain are within the unit circle to guarantee the steady of the proposed D-LDO system under the heavy and light load conditions.

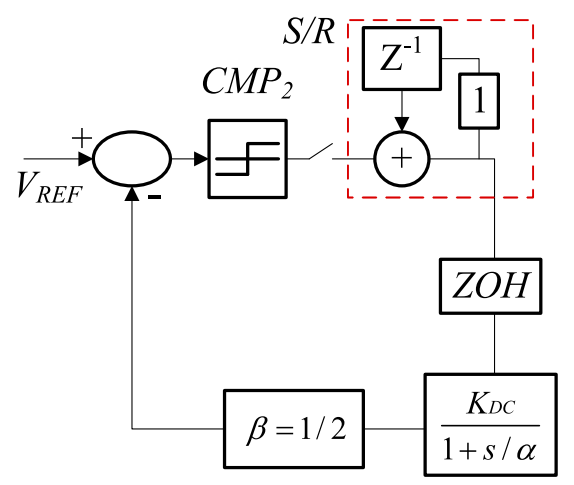

(a)

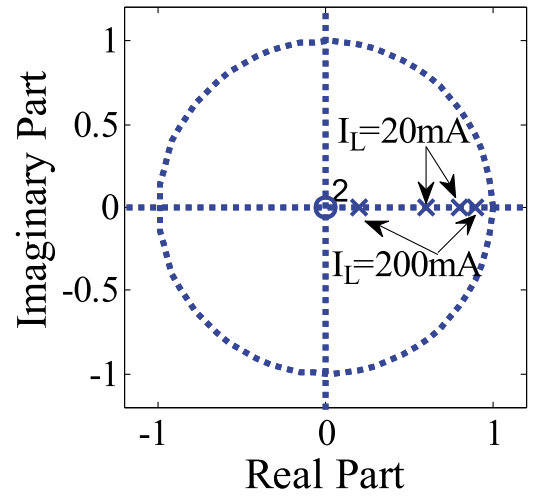

(b)

Fig. 7. (a) Small signal model and (b) root loci of proposed D-LDO under the conditions of heavy load $\left(\mathrm{I}_{\mathrm{L}}=200 \mathrm{~mA}\right)$ and light load $\left(\mathrm{I}_{\mathrm{L}}=20 \mathrm{~mA}\right)$.

\section{Simulated results}

The proposed D-LDO has been designed and simulated in the SMIC $65 \mathrm{~nm}$ process with $1.2 \mathrm{~V}$ supply and $1.0 \mathrm{~V}$ output voltage. The HSIM is chosen as the SPICE simulator. According to the transistor level simulation results shown in Fig. 8, the output overshoot and undershoot voltages are 27 and $26 \mathrm{mV}$, respectively, with load steps of 20 to $200 \mathrm{~mA}$ with a 10-ns edge time, while they are 183 and $1363 \mathrm{mV}$, respectively, when the push-pull feedback loop is not engaged. On the other hand, with the push-pull feedback loop control, the steady voltage ripples are not degraded, which are simulated to be 15 and $7 \mathrm{mV}$ under 20 and $200 \mathrm{~mA}$ load current, respectively. And the setting time is approximately $1.25 \mathrm{~ms}$. In addition, the quiescent current is only $400 \mu \mathrm{A}$ with maximum load current up to $200 \mathrm{~mA}$. When $\mathrm{I}_{\mathrm{L}}=200 \mathrm{~mA}$, the current peak efficiency is $99.8 \%$. The output capacitance is $1 \mu \mathrm{F}$.

With the help of Layout XL in Virtuoso, the estimated layout area is around $0.42 \mathrm{~mm}^{2}$ except for output capacitor.

The proposed D-LDO is compared with prior arts, as shown in Table II. The output voltage variation $\left(\Delta \mathrm{V}_{\text {OUT }}\right)$ enhancement over previous work is notable. In order to compare the key performances of the proposed D-LDO with other state-ofart designs, the figure-of-merit for response time $\left(\mathrm{FOM}_{\mathrm{RESP}}\right)$ presented in [14] is employed. A larger $\mathrm{FOM}_{\mathrm{RESP}}$ implies faster response performance. 


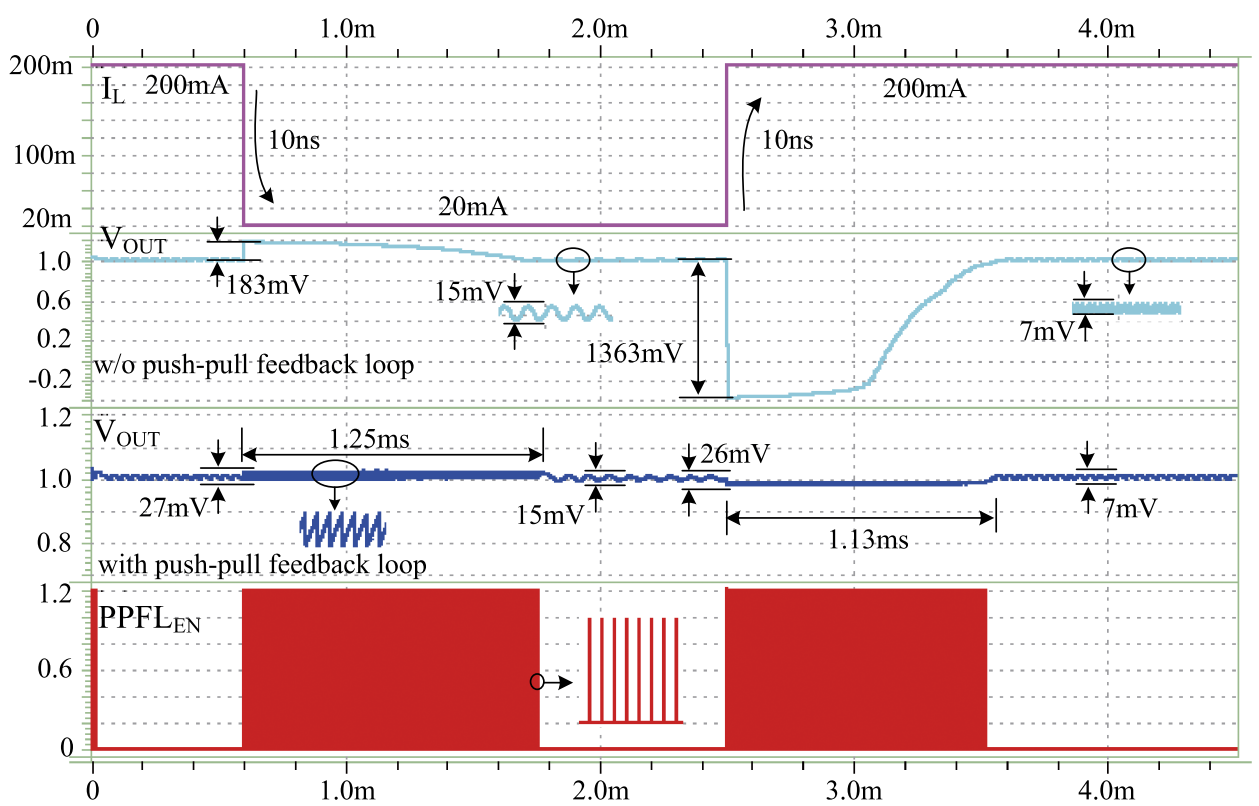

Fig. 8. Simulated load transient response.

Table II. Comparison of performance

\begin{tabular}{|l|c|c|c|}
\hline & {$[2]$} & {$[9]$} & This work \\
\hline Technology $(\mathrm{nm})$ & 130 & 180 & 65 \\
\hline Active area $\left(\mathrm{mm}^{2}\right)$ & $\mathrm{N} / \mathrm{A}$ & 0.81 & $\approx 0.42$ \\
\hline Input voltage $(\mathrm{V})$ & 1.0 & $0.9-1.8$ & 1.2 \\
\hline Output voltage $(\mathrm{V})$ & 0.8 & $0.8-1.5$ & 1.0 \\
\hline Maximum load current $(\mathrm{mA})$ & 100 & 200 & 200 \\
\hline Output Capacitor & $0-100 \mathrm{pF}$ & $1 \mu \mathrm{F}$ & $1 \mu \mathrm{F}$ \\
\hline Quiescent current $(\mu \mathrm{A})$ & $1.8-67$ & 750 & 400 \\
\hline Peak current eff. $(\%)$ & $99.93^{(a)}$ & 99.6 & 99.8 \\
\hline Edge time (ns) & 300 & 500 & 10 \\
\hline Load step (mA) & $0.1-100$ & $1-100$ & $20-200$ \\
\hline$\Delta V_{\text {Out }}(\mathrm{mV})$ & 80 & 70 & 27 \\
\hline FOM ${ }_{\text {RESP }}^{(c)}$ & 24.9 & 1.62 & 32.7 \\
\hline
\end{tabular}

(a) Calculated by using maximum load and quiescent current.

(c) $\mathrm{FOM}_{\mathrm{RESP}}=\mathrm{I}_{\mathrm{STEP}} /\left(\mathrm{I}_{\mathrm{MIN}} \times \Delta \mathrm{V}_{\mathrm{OUT}} \times \mathrm{T}_{\mathrm{EDGE}}\right)$ is shown in [14].

\section{Conclusions}

This paper presents a novel D-LDO with voltage peak detecting and push-pull feedback loop operation. The proposed D-LDO operates at $1.2 \mathrm{~V}$ supply and $65 \mathrm{~nm}$ technology. The simulation results show that the proposed D-LDO's transient response performance has been significantly improved owning to the proposed voltage peak detecting technique and push-pull feedback loop control. In addition, the proposed D-LDO achieves maximum load capability up to $200 \mathrm{~mA}$.

\section{Acknowledgments}

The authors would like to thank the supports from the General Program of National Natural Science Foundation of China (Grant No. 61471354). 